\title{
Anxiety selectively increases information-seeking in response to large changes
}

Caroline Charpentier*1-2 ${ }^{*}$ Irene Cogliati Dezza*3-5, Valentina Vellani*3-4 ${ }^{*}$ Laura Globig ${ }^{1-2}$, Maria Gädeke ${ }^{6}$, Tali Sharot ${ }^{3-4}$

\author{
Affiliations: \\ ${ }^{1}$ Division of Humanities and Social Sciences, California Institute of Technology, Pasadena, \\ CA, USA. \\ ${ }^{2}$ Institute of Cognitive Neuroscience, University College London, London, UK. \\ ${ }^{3}$ Department of Experimental Psychology, University College London, London, WC1H 0AP, \\ UK. \\ ${ }^{4}$ The Max Planck UCL Centre for Computational Psychiatry and Ageing Research, \\ University College London, London, WC1B 5EH, UK \\ ${ }^{5}$ Department of Experimental Psychology, Ghent University, B-9000 Ghent, BE \\ ${ }^{6}$ Division of Medical Psychology, University of Bonn, 53113 Bonn, Germany \\ *These authors contributed equally.
}




\begin{abstract}
Seeking information when anxious may help reduce uncertainty and guide decision-making. If information is negative or confusing, however, this may increase anxiety further. Information gathered under anxiety can thus be beneficial and/or damaging. Here, we examine whether anxiety leads to a general increase in information-seeking, or rather to situation-dependent changes in the type of information sought. In two controlled laboratory studies, we show that both trait and induced anxiety selectively increased information-seeking. In particular, anxiety did not enhance the general tendency to seek information, nor did it alter the valence of the information gathered. Rather anxiety increased information-seeking specifically in response to large changes in the environment. This was true even when the cause of the anxiety was not directly related to the information sought. As anxious individuals have been shown to have problems learning in changing environments, greater information-seeking in such environments may be an adaptive compensatory mechanism.
\end{abstract}

\title{
Significance statement
}

What people know is partially shaped by what information they choose to seek out. Information in turn, guides people's actions, and influences their emotions and cognition. Here, we show that the decision to seek out information is significantly altered by participants' mental state. In particular, when anxious, the willingness to pay for information increases selectively in response to large changes in the environment. Such modulation could be an adaptive compensatory mechanism, as anxiety has been previously associated with impairments in learning in changing environments. 


\section{Introduction}

Gathering information can be an adaptive response to feeling anxious. This is because information can increase one's sense of control, reduce an aversive sense of uncertainty, and help guide action (for review Sharot \& Sunstein, 2020). However, if the information revealed is negative or confusing, it can further increase anxiety (Johnston \& Davey, 1997; Norr et al., 2014; Shabahang et al., 2020; Dörnemann et al., 2021). Thus, the information people seek out when anxious could have a beneficial or a damaging effect on their well-being. Here, we examine whether and how anxiety alters the features of information people seek out.

While anxiety has been shown to have widespread effects on learning and decision-making (Browning et al, 2015; Hauser et al, 2017; Huang et al, 2017; Hein et al, 2021), its potential effect on the very first stage of the learning, namely the decision to seek information, has been relatively understudied. There is evidence that when people are anxious about a specific topic or event, such as health or politics, they search for more information about that topic/event (McMullan et al, 2019; Baugmartner \& Hartmann, 2011, Dillard et al, 2020; Drouin et al, 2020, Gadarian \& Albertson, 2014). However, anxiety can provide a global, rather than specific, danger signal. We thus hypothesized that the effects of anxiety on what people want to know may be observed even when the reason for anxiety is unrelated to the information sought (e.g., anxiety triggered by a professional conflict may impact information-seeking about finance or health).

We test three non-mutually exclusive hypotheses of how anxiety alters information seeking. First, we test whether anxiety is related to an increase in the general frequency of informationseeking. As knowledge can enhance a person's sense of control, which is reduced in anxiety (Gallagher et al. 2014), greater information-seeking may be a compensatory mechanism, regardless of the specific cause of the anxiety. Second, we test whether anxiety is related to the valence of information people seek out. On one hand, anxious individuals are known to exhibit attentional biases towards negative stimuli (MacLeod and Mathews, 1988, Bar-Haim et al., 2007; Cisler and Koster, 2010), suggesting that anxiety may increase the search for negative (over positive) information. On the other hand, anxious individuals need less negative information to reach negative conclusions than vice versa (Globig et al., 2020; Garrett et al., 2018). Thus, they may seek negative information less than positive information. Third, we test whether anxiety is related to greater information-seeking specifically when large changes in the environment occur. Anxious individuals have problems learning in changing environments (Browning et al, 2015; Hein et al, 2021), thus they may need more information in such instances.

To test these hypotheses, we conducted three studies. First, we provide a conceptual replication of the existing literature, which suggest that when people are anxious about a specific topic they search for more information about that topic (Study 1). In particular, we test whether COVID-19 anxiety is associated with greater information-seeking about COVID-19. Next, we conducted two control laboratory experiments (Study 2) to examine whether and how anxiety 
effects information-seeking when information is unrelated to the cause of the anxiety. We were interested in effects on (i) the frequency of information-seeking, (ii) the valence of information sought, and (iii) whether effects were observed selectively in response to large changes in the environment. In one experiment (Study 2a) we examined associations between these factors and trait anxiety, while in the second experiment (Study 2b) we manipulated anxiety, thus testing for a causal link between anxiety and the above aspects of information-seeking. By dissociating the cause of the anxiety from the content of the information sought, we provide a broad perspective on the effects of anxiety on information-seeking.

\section{Methods}

\section{Study 1: Information-seeking and anxiety in a threatening natural setting}

\section{Participants}

\section{Time point 1}

1166 participants took part in the study by completing an online questionnaire on Prolific Academic (https://www.prolific.co/) between March 26-29, 2020. The sample was representative of the US population in terms of age, sex and ethnicity (83\% Caucasian, 6\% African-American, 5\% Asian, 4\% mixed, 1\% other). All participants were residing in the US at the time of testing. Participants' engagement and attention were tested through various catch trials throughout the experiment (more details are provided in the supplementary material). 21 participants failed to select the appropriate response more than once on catch trails and therefore were excluded from the analysis. 101 participants did not indicate their ethnicity, so the final sample was composed of 1065 individuals (age=44.78, $\mathrm{SD}=15.49$; females $=51.8 \%$ ). All participants were paid $£ 3.75$ for their participation. The study was approved by the departmental ethics committee at UCL.

\section{Time point 2}

Between April 23-25 2020, 700 of the participants recruited in Time point 1 filled in a second questionnaire on Prolific Academic (age $=46.3, \mathrm{SD}=15.22$; females=51.9\%). No participant failed more than one catch trial (more details are provided in the supplementary material). Participants were paid $£ 1.88$ for their participation. The study was approved by the departmental ethics committee at UCL.

\section{Procedure}

\section{Time point 1}

Participants provided an answer to this question: "How often do you consume information on COVID-19 (news, internet etc..)?" on a scale from "Never" (0) to "At least once an hour" (6) on a discrete scale (See Supplementary Materials for details). State anxiety was measured with 
the Short State Anxiety Inventory (SSAI, Marteau \& Bekker, 1992). We also assessed COVID19-related anxiety by asking participants to answer on a continuous visual analogue scale ranging from 0 (none at all) to 100 (very much) the following questions: 1) "Are you anxious about your own health?"; 2) "Are you anxious about the health of your loved ones in light of COVID-19?"; 3) "Are you anxious about dealing with lockdown in your area?"; 4) "Are you anxious about the consequence to your income/savings in light of COVID-19?"; 5) "Are you anxious about not being able to exercise?"; 6) "Are you anxious about not having access to food/medicine/other supplies?"; 7) "Are you anxious about not being able to socialize?".

We collected demographic information including participants' age, gender, current residence, level of education, household income, political orientation, ethnicity, whether they had dependents and the satisfaction with their health insurance. Additional information collected was part of other studies carried out by members of our lab (e.g., Globig et al., 2020, Blain et al., 2021). We report the other questions included in the survey in the Supplementary Material.

\section{Time point 2}

Participants filled in the same questions as in Time point 1. Many of the items presented in the survey completed by subjects in Time Point 1 for the parallel studies conducted in the lab were not included at Time Point 2 (See the Supplementary Materials for details). Demographics were not assessed a second time.

\section{Analysis}

To examine whether COVID-related information consumption was associated with anxiety we computed an 'Anxiety Index Score' by summing the COVID-19-related anxiety questions and the SSAI score (the SSAI score was considered as one measure) and dividing by the total number of questions. The SSAI scores were transformed to the same range as the COVID-19related anxiety questions (0-100). Then, we ran a linear regression predicting COVID-related information-seeking ratings from the Anxiety Index Score, adding all demographics as covariates (age, gender, educational level, income, political orientation, ethnicity, whether they had dependents and the satisfaction with their health insurance). We ran the same analysis with information-seeking rating as the dependent measure, but with either the average of the COVID-19-related anxiety questions or the SSAI score separately, with all demographics as covariates. All the analyses were repeated separately for time 1 and 2. Group-level linear regressions were carried out with $\mathrm{R}$ (4.0.1) using the $1 \mathrm{~m}$ package.

\section{Study 2}

\section{Study 2a: The relationship between information-seeking and trait anxiety}

\section{Participants}

All participants were recruited via the UCL subject pool, and the experiment was approved by the departmental ethics committee at UCL. 45 participants were recruited, but data from participants who missed more than $25 \%$ of all trials were excluded $\left(\mathrm{N}_{\text {excluded }}=3\right)$. Final sample was then of 42 participants ( 15 males, 27 females, mean age $=28.02$ years \pm 11.62 (SD), age 
range: 18 to 66 years). This sample size is consistent with previous studies that have investigated learning and decision-making in relation to trait anxiety (Browning et al., 2015; Charpentier et al., 2016; Fung et al., 2019; Norr et al., 2014; Xu et al., 2013). At the end of the task, participants completed the State-Trait Anxiety Inventory (STAI). Mean trait anxiety was $42.21 \pm 11.69$ (SD) and ranged from 20 to 68 . Some of the task-related data was previously published in Charpentier et al, 2018 - the relationship between information-seeking tendencies and trait anxiety however had never been assessed before in that dataset nor previously published.

\section{Procedure}

Participants played a Stock Market Task (Charpentier et al., 2018; Vellani et al., 2020; Figure 2). This task consisted of 4 blocks of 50 trials each. At the beginning of each block, participants were endowed with 100 points, worth £10, which they were to invest in 2 of 5 fictitious stocks that compose a 'global market'. On each trial participants observed the global market evolution (a dynamic increase or decrease in the curve lasting 2.3s). Given that the participant's portfolio consisted of 2 companies out of the 5, the global market was a partial indicator of the change to the participant's own portfolio value. Unbeknown to the participants, on each trial there was a $65 \%$ likelihood that their portfolio would follow the market trend; otherwise, the portfolio would vary in the opposite direction than the market with a randomly-generated magnitude.

On each trial, after observing the change to the global market, participants were given the opportunity to find out their portfolio value (Figure 2). In particular, they had $8 \mathrm{~s}$ to indicate how much they were willing to pay to receive this information or to avoid it. They did so using a scale ranging from $99 p$ to avoid information, through 0 , to $99 p$ to receive information (left/right positions of 'YES' information and 'NO' information were counterbalanced across participants). We refer to this scale as the Willingness To Pay (WTP) scale (Becker et al, 1964). Participants were told that the more they were willing to pay the greater the probability that their decision (to receive or avoid information) would be honored. If they selected ' 0 p', then information was delivered at random (50\%). If they selected an amount between $1 \mathrm{p}$ and $20 \mathrm{p}$, their wish was honored with $55 \%$ probability; between $21 \mathrm{p}$ and $40 \mathrm{p}$, their wish was honored with $65 \%$ probability; and so on up to $95 \%$ probability. Participants were not aware of how exactly each WTP response was converted in the probability of receiving information. Next, their portfolio value in points was presented on screen for $3 \mathrm{~s}$ or hidden ('XX points' was shown).

At the end of the task one trial was selected at random (regardless of whether information was delivered) for payment. Participants would receive their portfolio value on that trial. If they paid money to receive or avoid information on that trial and their choice was respected that amount was deducted from their payment (e.g. if they earned 110 points and selected to pay $£ 0.50$ to receive information which they did, then they will get $11-£ 0.50=£ 10.50$ ).

In the last two blocks (blocks 3-4) after they observed the global market participants were asked to answer two additional questions which are beyond the scope of this study (more details in the Supplementary Materials). 


\section{Analysis}

First, we computed willingness to pay (WTP) on every trial, with amount paid to avoid information scored negatively, and amount paid to receive information positively (zero is simply coded as zero).

We then investigated how trait anxiety was related to information-seeking behavior. We first performed a group-level linear regression to predict a participant's average WTP from trait anxiety score, controlling for age and gender. We then focused on whether specific factors that drive information-seeking were modulated by trait anxiety. To do so, we performed individuallevel linear regressions to predict, for each participant, WTP for information on each trial from two factors (z-scored) we have previously shown to impact information-seeking in this task (Charpentier et al., 2018, Vellani et al., 2020): (i) valence (quantified as signed market change, which is the amount by which the market went up or down) and (ii) absolute market change, which represents the magnitude of changes in the environment, independent of valence. The resulting betas were then compared to zero with a two-tailed one sample t-test (Figure 3). We then performed two separate group level linear regressions predicting each beta coefficient from trait anxiety, controlling for age and gender. Group-level linear regressions were carried out with R (4.0.1) using the lm package, while individual-level linear regressions were carried out with Matlab (R 2019a) using the function glmfit.

\section{Study 2b: Induced anxiety and information-seeking}

\section{Participants}

Fifty participants were recruited via the UCL subject pool and the experiment was approved by the departmental ethics committee at UCL. One participant did not complete the experiment and data from another participant was lost. Final data are thus reported on 48 participants. The anxiety-induced group was composed of 24 participants (10 males, 14 females, mean age $=21.96$ years \pm 3.51 (SD), age range: 19 to 32 years) and the control group of 24 participants (10 males, 14 females, mean age $=23.46$ years \pm 4.79 (SD), age range: 19 to 34 years). There was no age difference between groups $(\mathrm{t}(46)=1.238, \mathrm{p}=0.22)$. The sample size was consistent with previous studies that have used anxiety induction procedures during learning and decisionmaking tasks (Garrett et al, 2018; Hein et al., 2021; Vogel et al., 2018), and with the sample size of Study 2a.

\section{Procedure}

Participants performed the same task as in Study 2a (Figure 2).

\section{Induced anxiety}

Before the task, participants were randomly assigned to one of two groups: induced anxiety group and control group. The induced anxiety group was administered a modified version of the Trier Social Stress Test (TSST, Kirschbaum et al., 1993; Garrett et al., 2018; Birkett, 2011; Globig et al., 2021). Specifically, they were told that at the end of the task, they would have to give a 5-minute presentation on a surprise topic in front of a panel of senior academics and that they would be videotaped and judged during that presentation. The main difference with the typical TSST is that participants were threatened by a stressful social event and completed the 
task under that threat, but the threat was in fact never executed. In addition, they were given six difficult math problems to solve in less than 30s before the beginning of the task. Participants assigned to the control group were told they would have to write a short essay at the end of the task, but would not be judged. They were also given six very easy math problems to solve in 30s. To measure whether the manipulation was effective, all participants completed the Short State Anxiety Inventory (SSAI, Marteau \& Bekker, 1992) before and after the manipulations described above. We then used these questionnaires to compute the change in anxiety levels by subtracting the anxiety score reported before the stress or control manipulation from the anxiety score reported after the manipulation. Using change in SSAI as a measure of task-induced anxiety allows individual variations in response to the TSST to be taken into consideration, as it is known that responses to the TSST vary widely among individuals depending on age (Kudielka et al., 2007), gender (Kirschbaum et al., 1992), education (Fiocco et al., 2007), personality traits (Oswald et al., 2006), use of nicotine, alcohol and caffeine (Kudielka et al., 2007) and use of medications (Freis el.,2006; for an overview of individual differences in TSST performance, see Kudielka et al., 2009).

\section{Analysis}

Analyses was exactly as in Study 2a, but with change in Short State Anxiety Inventory scores instead of trait anxiety scores. 


\section{Results}

\section{Study 1. Anxiety is associated with increased information-seeking about COVID-19.}

To investigate how anxiety affects information-seeking in a 'real world' threatening event, 1065 participants filled in an online questionnaire at the beginning of the COVID-19 pandemic (between March 26-29, 2020). Participants were asked "How often do you consume information on COVID-19 (news, internet etc..)?" on a scale from "Never" (0) to "At least once an hour" (6). Anxiety was measured using both the Short State Anxiety Inventory (SSAI, Marteau \& Bekker, 1992) and by asking participants to rate their anxiety related to COVID. In particular, the later we assessed by asking participants to rate their anxiety about their health, health of loved ones, dealing with lockdown in their area, the consequence of COVID to their income/savings, not being able to exercise, not having access to food/medicine/other supplies and not being able to socialize, on scale ranging from 0 (none at all) to 100 (very much). We used all the above anxiety-related responses to compute an Anxiety Index Score. This was obtained by summing the COVID-19-related anxiety questions and the SSAI score and dividing by the total number of questions (considering the SSAI score as one question). As detailed below, results are the same when using only SSAI or only COVID-related anxiety to measure anxiety levels. 700 of the participants answered the same questions again between April 23 to April 25, 2020.

To test the relationship between anxiety and self-reported information-seeking behavior, we ran a linear regression predicting self-report information-seeking about COVID-19 from the Anxiety Index Score. Age, gender, educational level, income, political orientation, ethnicity, dependents, and satisfaction with health insurance were all added as covariates. Results show that participants with higher Anxiety Index Score reported engaging more in informationseeking about the pandemic at Time point $1\left(\beta=0.009 \pm 0.001, p<0.001, f^{2}=0.045\right.$, Figure 1A) and Time point $2\left(\beta=0.008 \pm 0.002, \mathrm{p}<0.001, f^{2}=0.039\right.$, Figure 1B). The same results are also obtained when the average of the COVID-19-related anxiety questions was included as the only predictor of information-seeking (Time point $1: \beta=0.008 \pm 0.001, \mathrm{p}<0.001, f^{2}=$ 0.037; Time point 2: $\left.\beta=0.008 \pm 0.002, \mathrm{p}<0.001, f^{2}=0.035\right)$ or when the SSAI score was included as the only predictor (Time point $1: \beta=0.011 \pm 0.001, \mathrm{p}<0.001, f^{2}=0.05$; Time point 2: $\left.\beta=0.009 \pm 0.002, \mathrm{p}<0.001, f^{2}=0.028\right)$.

Results from Study 1 suggest that individuals who report higher state anxiety and higher anxiety about the pandemic are more likely to seek information related to the pandemic. 

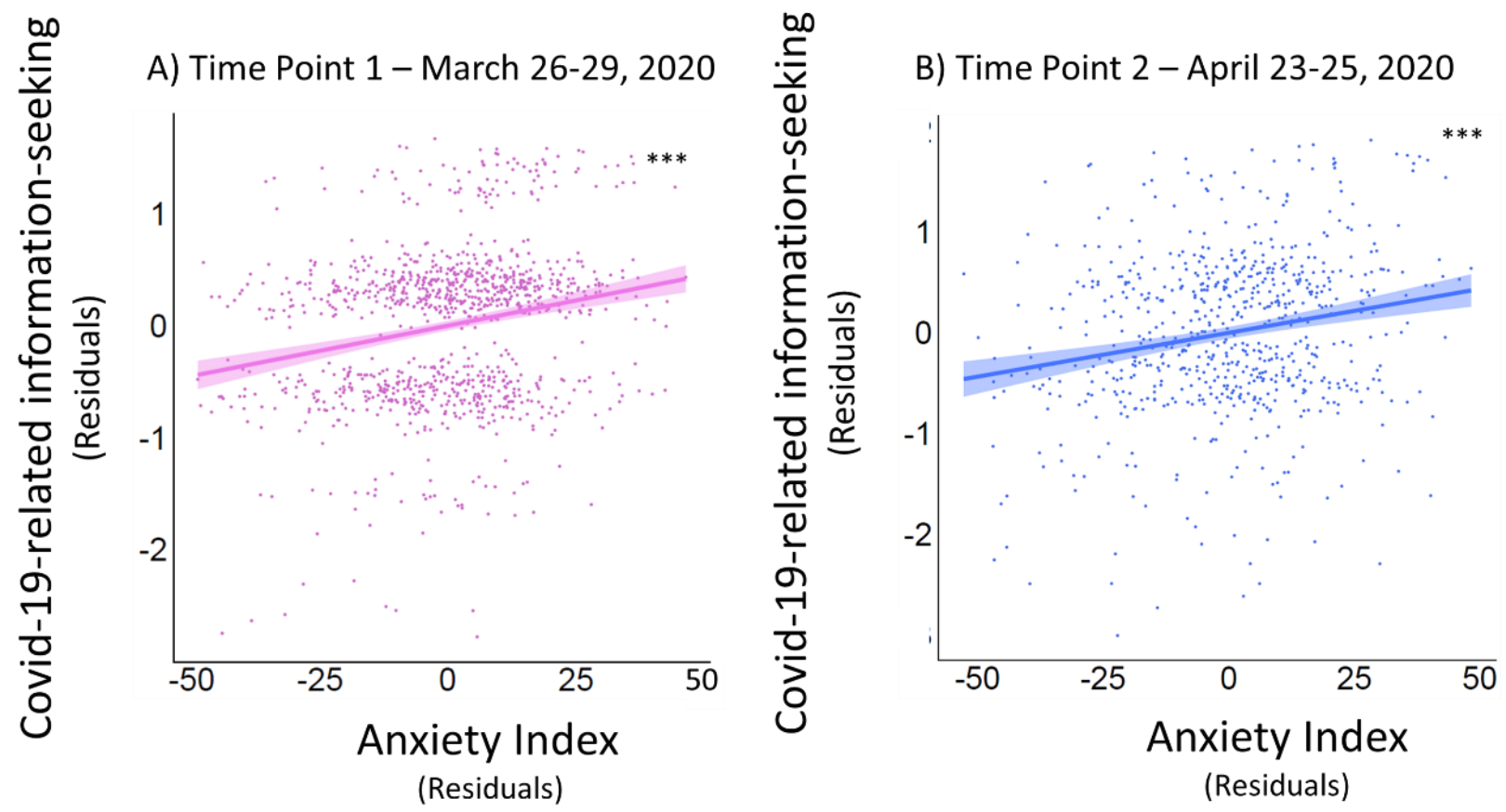

Figure 1. Individuals reporting greater Covid-19-related anxiety seek more Covid-19-related information (Study 1). Plotted are the partial linear regressions predicting self-reported Covid-19related information-seeking (obtained by asking participants "How often do you consume information on COVID-19 (news, internet etc..)?" on a scale from "Never" (0) to "At least once an hour" (6) on a discrete scale) from the Anxiety Index Score (obtained by summing the COVID-19-related anxiety questions and the SSAI score and dividing by the total number of questions) at Time point 1 (A; $\mathbf{p}<$ $\mathbf{0 . 0 0 1})$ and Time point $2(\mathbf{B} ; \mathbf{p}<\mathbf{0 . 0 0 1})$, controlling for age, gender, level of education, household income, political orientation, ethnicity, whether they had dependents and the satisfaction with their health insurance. Both graphs show that individuals with higher Anxiety Index Score report engaging more in COVID-related information seeking. $* * * p<0.001$. (Note that the observed clustering along the Y-axis in both graphs is due to the discrete nature of the rating scale used to measure Covid-19related information-seeking.

\section{Study 2a Trait anxiety is selectively associated with increased information- seeking in response to large changes.}

The aim of Study 2a was to test whether anxiety is associated with an increase in informationseeking even when the information sought is unrelated to the source of anxiety, and to test whether such an increase is valence specific and/or observed selectively in response to large changes.

To that end, 45 participants performed an information-seeking task adapted from our previous publications (Charpentier et al 2018; Vellani et al 2020; Figure 2). Three participants were excluded for missing more than $25 \%$ of trials; therefore, data of 42 subjects was analyzed. At the beginning of each of the four blocks, participants were endowed with $£ 5$ to invest in two of five stocks in a simulated stock market. There were 50 trials per block. On each trial, 
participants observed the evolution of the market (i.e. whether the market was going up or down) and the exact value of the market. They then bid for a chance to know (or remain ignorant about) the value of their portfolio. Specifically, they indicated how much they were willing to pay to receive or avoid information about the value of their portfolio on a scale ranging from $99 \mathrm{p}$ to gain knowledge through $0 \mathrm{p}$ (no preference) to $99 \mathrm{p}$ to remain ignorant. The more they were willing to pay, the more likely their choice was to be honored. Altering the direction and magnitude by which the market was changing from trial to trial allowed us to manipulate both the expected valence of the information (likely positive gain or negative loss) and the degree of change. Information was non-instrumental; it could not be used to increase rewards, avoid losses, or make alterations to portfolio. At the end of the task, participants completed the State-Trait Anxiety Inventory (STAI).

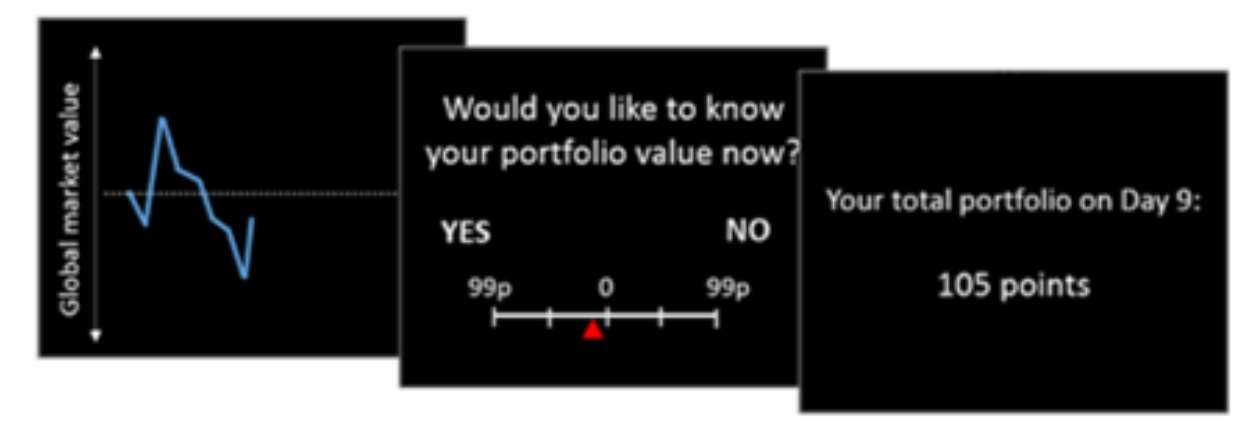

Figure 2. Stock Market Task. Participants observed the market going up or down on each trial. They were then asked whether they want to find out the value of their portfolio and had $8 \mathrm{~s}$ to indicate this using a willingness to pay scale. Specifically, the more they paid towards 'YES' the more likely they would be to receive information, and the more they paid towards 'NO' the more likely they would remain ignorant. They then receive feedback for $3 \mathrm{~s}$ with either the value of their portfolio or ' $\mathrm{XX}$ points'.

\section{Trait anxiety is not associated with increased frequency of information-seeking.}

We first tested whether trait anxiety was related to a general increase in information-seeking by examining whether the Willingness To Pay (WTP) for information was related to anxiety levels. WTP was coded positively if participants indicated they wanted to receive information and negatively if they wanted to avoid information. We ran a linear regression to predict participants' average WTP from trait anxiety scores controlling for age and gender. Results show that anxiety levels did not predict WTP $\left(\beta=0.039 \pm 0.23, \mathrm{p}=0.863, f^{2}=0.001\right.$; the same result emerges when excluding the one outlier with very negative WTP: $\beta=0.087 \pm 0.154$, $p$ $=0.574, f^{2}=0.009$; Figure 3). This suggests that trait anxiety is not related to general changes in information-seeking. 


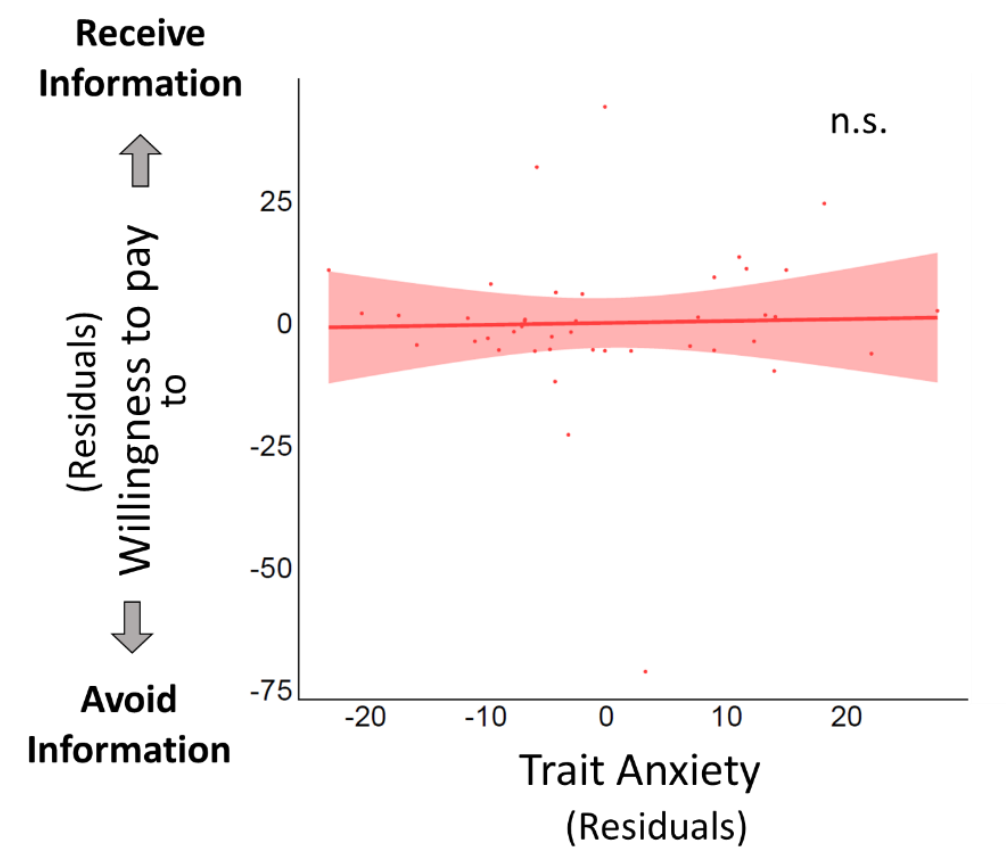

Figure 3. Trait anxiety is not related to willingness to pay for information (Study 2a). Plotted is the partial linear regression predicting WTP (in pence, coded positively if participants indicated they wanted to receive information and negatively if they wanted to avoid information) from trait anxiety, controlling for age and gender $(\mathrm{p}=0.863)$. Results do not change when removing the one outlier. Also note that plotted values are residuals after controlling for age and gender, hence the negative trait anxiety values.

Trait anxiety is associated with greater information-seeking in response to large changes We have previously shown that two features significantly impact WTP for information (Charpentier et al., 2018 \& Vellani et al., 2020). The first is the expected valence of the information (quantified as signed market change, which is the amount by which the market went up or down), with participants wanting information more when the market was going up and thus the likelihood for gain was greater. The second was absolute market change (signaling large or small changes in the market regardless of direction), with participants wanting information more when there were large changes in the market.

A linear regression was conducted for each participant to predict WTP from the signed market change and absolute market change (z-scored) and the resulting betas were then compared to zero with a one sample t-test. Consistent with previous studies (Charpentier et al., 2018; Vellani et al. 2020), we found that participants' WTP for information was greater when the market was going up rather than down (signed market change: $\beta=1.23 \pm 0.56, \mathrm{t}(41)=2.17, \mathrm{p}=0.035$ ) and when there were large changes in the market (absolute market change: $\beta=1.58 \pm 0.46$, $\mathrm{t}(41)=3.46, \mathrm{p}=0.001 ;$ Figure 4A). 
B)

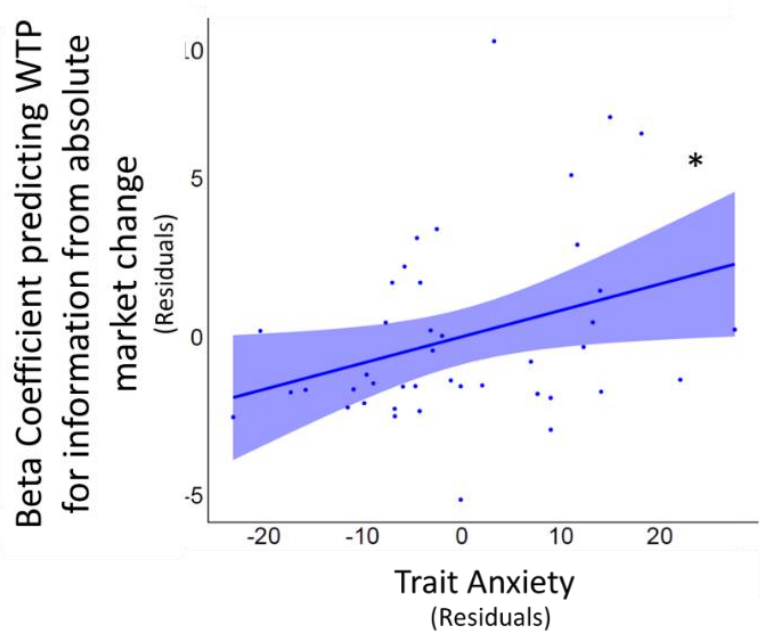

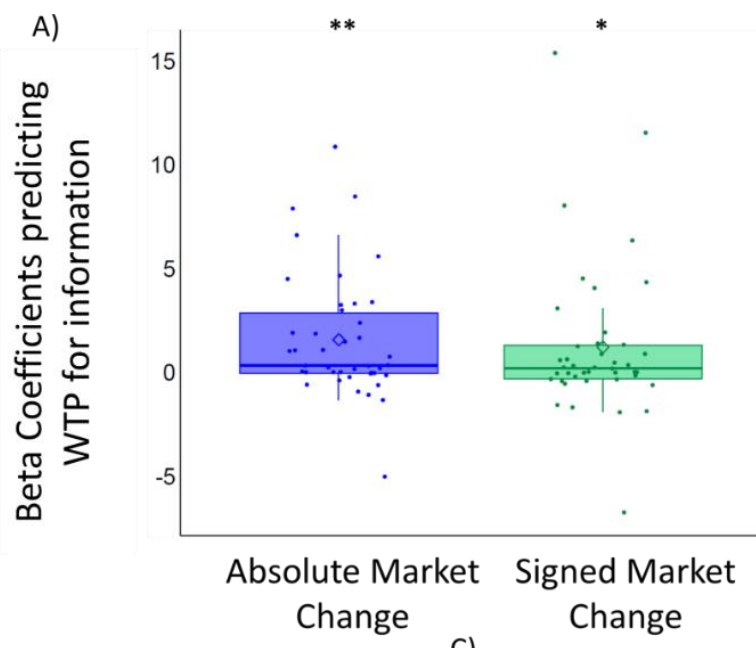

C)

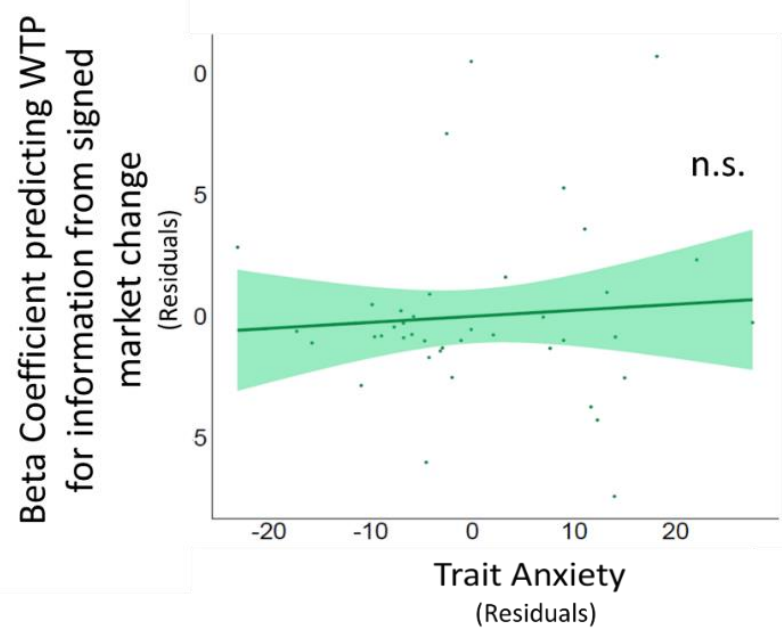

Figure 4. Increased trait anxiety is associated with greater information-seeking when there are large changes in the environment regardless of valence (Study 2a). (A) A linear regression was conducted for each participant to predict WTP from the signed market change and absolute market change and the resulting betas were than compared to zero with a one sample t-test. Both signed market change (signaling whether the market was going up or down) and absolute market change (signaling whether there were large or small changes in the market) predicted participants' willingness to pay for information. Specifically, participants' willingness to pay for information was greater when the market was going up rather than down and when there were large changes in the market. Plotted are the beta coefficients reflecting the effect of signed (green) and absolute (purple) market change on WTP. Horizontal lines indicate median values, boxes indicate $25-75 \%$ interquartile range, diamonds indicate mean values and whiskers indicate $1.5 \times$ interquartile range; individual coefficients are shown separately as dots. $* * p=0.01, * p<0.05$. (B-C) Increased trait anxiety is associated with greater impact of absolute market change on information-seeking $(\mathrm{p}=0.038)(\mathbf{B})$ but not with changes to the impact of valence on information-seeking $(\mathrm{p}=0.615)(\mathbf{C})$. (B-C) Plotted are the partial linear regressions predicting the beta coefficients shown in (A) from trait anxiety, controlling for age and gender. Also note that in panels B and C plotted values are residuals after controlling for age and gender, hence the negative trait anxiety values.

We then performed two linear regressions to predict each of the two beta coefficients (signed and absolute market change) from trait anxiety scores, controlling for age and gender. Results revealed that greater trait anxiety was related to an increase in the WTP for information when 
there were large changes in the market $\left(\beta=0.08 \pm 0.04, p=0.039, f^{2}=0.108\right.$; Figure 4B), and was unrelated to the impact of valence on information-seeking $\left(\beta=0.025 \pm 0.05, \mathrm{p}=0.616, f^{2}\right.$ $=0.007$; Figure 4C). Overall, results from Study 2a suggest that individual differences in trait anxiety are related to differences in the desire for information when there are large changes in the environmental. Anxiety was not associated with the frequency of information-seeking per $s e$, nor with valence-dependent information-seeking. Higher trait anxiety appears to be selectively associated with increased information-seeking in response to large changes in the environment.

In the next experiment, we replicated our findings using induced anxiety instead of trait anxiety, and investigated whether this relationship is causal by manipulating individuals' anxiety levels and measuring their information-seeking behavior.

\section{Study $2 \mathrm{~b}$. Anxiety leads to greater information-seeking in response to large changes}

To test for a causal relationship between anxiety and information-seeking, we manipulated anxiety levels of 50 participants using a variation of the Trier Social Stress Test, immediately prior to having them complete the Stock Market Task (see Methods for details). Data from 2 subjects were lost, therefore data from 48 subjects were analyzed. Before the task, participants were randomly assigned to one of two groups: induced anxiety group $(\mathrm{N}=24)$ and control group $(\mathrm{N}=24)$. The induced anxiety group was administered with a modified version of the Trier Social Stress Test (TSST, Kirschbaum et al., 1993; Garrett et al., 2018; Birkett, 2011; Globig et al., 2021). Specifically, they were told that at the end of the task, they would have to give a 5-minute presentation on a surprise topic in front of a panel of senior academics and that they would be videotaped and judged during that presentation. In addition, they were given six difficult math problems to solve in less than 30s before the beginning of the task. Participants assigned to the control group were told they would have to write a short essay at the end of the task, but would not be judged. They were also given six very easy math problems to solve in 30s. Participants completed the Short State Anxiety Inventory (SSAI, Marteau \& Bekker, 1992) before and after the manipulations described above.

\section{Anxiety levels were successfully manipulated}

The manipulation was successful. After the manipulation, individuals in the induced anxiety group exhibited greater anxiety, as measured by the SSAI scores $(\mathrm{N}=24$, mean SSAI score $=$ $16.42, \mathrm{SD}=4.35)$, than individuals in the control group $(\mathrm{N}=24$, mean SSAI score $=10.46$, $\mathrm{SD}=4.00)$ (difference: $\mathrm{t}(46)=4.93, \mathrm{p}<0.001$ ), with no difference between groups before the manipulation (induced anxiety group mean SSAI score $=9.79, \mathrm{SD}=3.93$; control group mean SSAI score $=10.08, \mathrm{SD}=3.78$; group difference: $\mathrm{t}(46)=0.26, \mathrm{p}=0.794)$. The change in reported anxiety (after minus before the manipulation) was significantly higher in the induced anxiety group compared to the control group (induced anxiety group mean SSAI score change= 
$6.63, \mathrm{SD}=3.83$, control group mean SSAI score change $=0.38, \mathrm{SD}=2.43$; group difference: $\mathrm{t}(46)=6.75, \mathrm{p}<0.001$, Figure 5).

There was substantial variability in individual susceptibility to the manipulation, with some individuals reporting increased anxiety after the control manipulation, and others individuals reporting very little change in anxiety after the anxiety manipulation (also see Kudielka et al., 2009 for a review; Kudielka et al., 2007; Kirschbaum et al., 1992; Oswald et al., 2006). To account for individual differences in response to the manipulation, and for consistency with Study 2a, we therefore used the change in SSAI as a predictor of information-seeking behavior in all analyses.

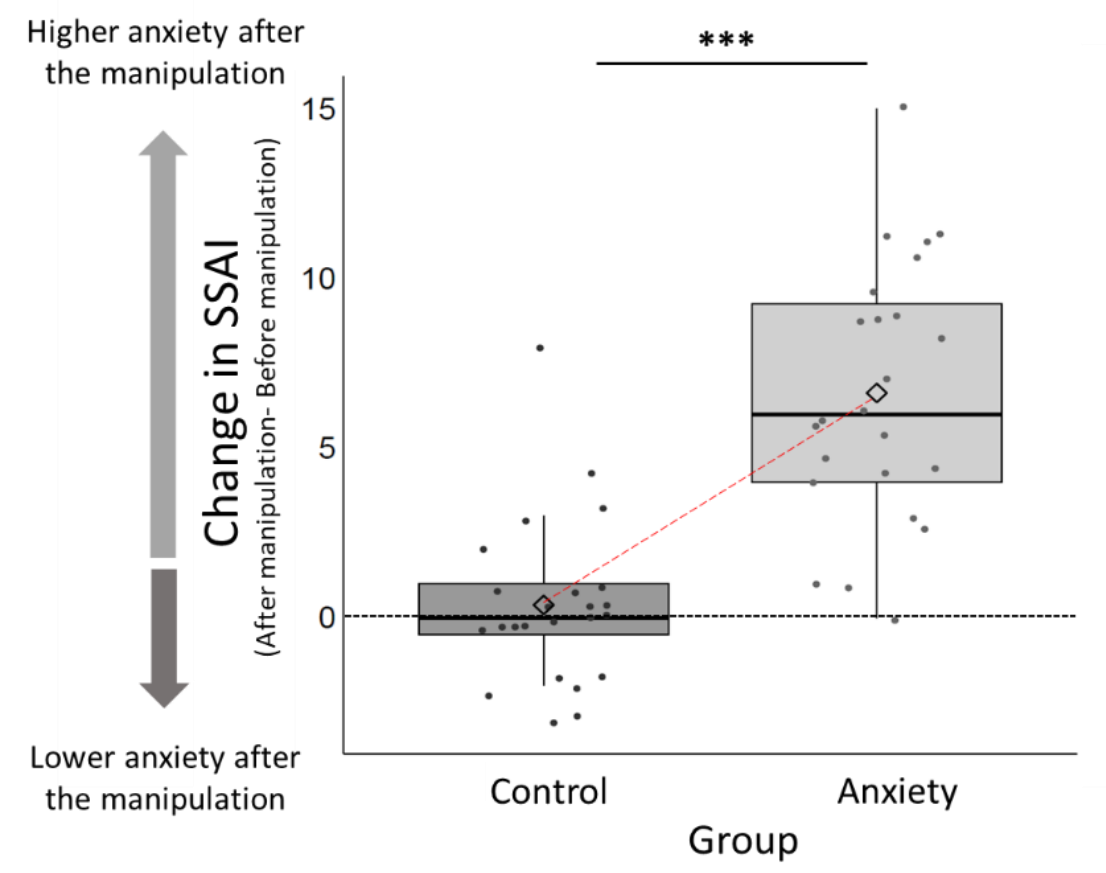

Figure 5. Change in anxiety levels was stronger in the induced anxiety group as compared to the control group (Study 2b). Plotted is the change in SSAI induced by the manipulation in both groups. Change in anxiety was computed for each individual by subtracting the SSAI score before the manipulation from the SSAI score after the manipulation. Horizontal lines indicate median values, boxes indicate $25-75 \%$ interquartile range, diamonds indicate mean values, the horizontal dotted line indicate 0 change in anxiety scores and whiskers indicate $1.5 \times$ interquartile range; individual scores are shown separately as dots. $* * * \mathrm{p}<0.001$.

\section{Induced anxiety did not alter overall willingness to pay for information.}

The results show that WTP for information was unrelated to induced anxiety. In particular, a linear regression predicting WTP from change in SSAI, controlling for age and gender was not significant $\left(\beta=0.320 \pm 0.32, \mathrm{p}=0.324, f^{2}=0.022\right.$; same results are obtained when excluding the one outlier: $\beta=0.112 \pm 0.196, p=0.573, f^{2}=0.007$; Figure 6). Consistent with results from Study $2 \mathrm{a}$, this result suggests that anxiety does not induce a general change in informationseeking. 


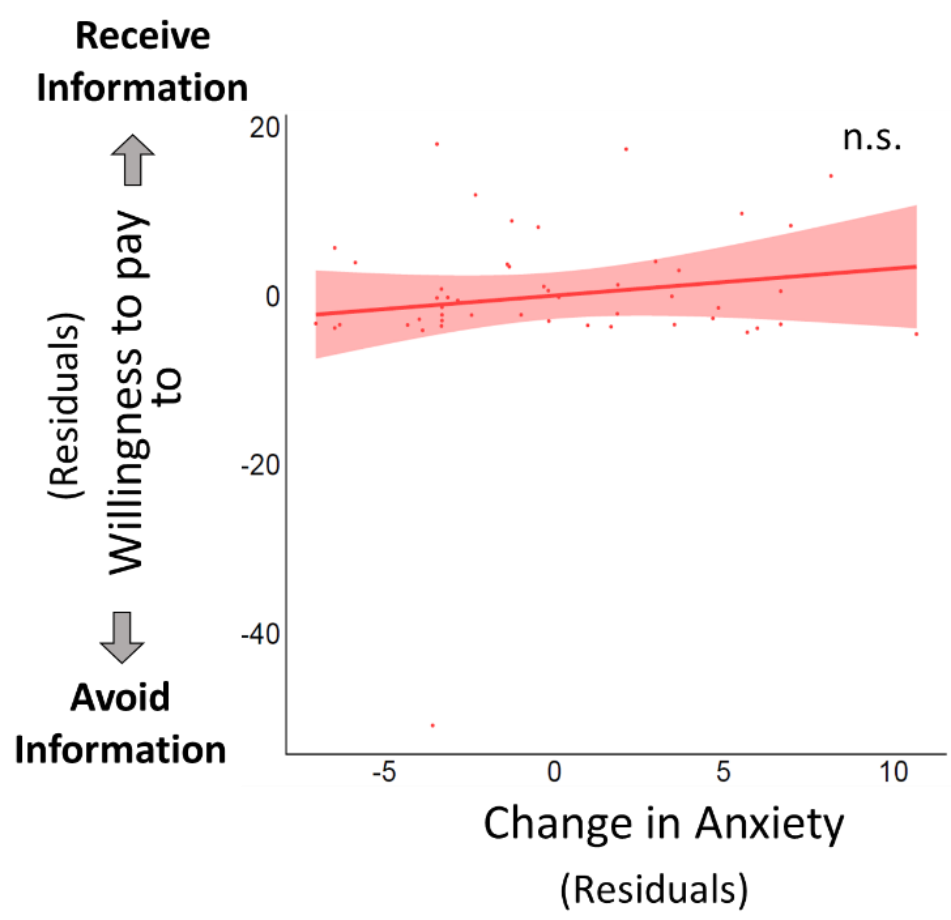

Figure 6. Change in anxiety is not related to willingness to pay for information (Study 2b). Plotted is the partial linear regressions predicting WTP (in pence, coded positively if participants indicated they wanted to receive information and negatively if they wanted to avoid information) from change in anxiety induced by the manipulation, controlling for age and gender $(\mathrm{p}=0.324)$. Results do not change when removing the one outlier.

\section{Induced anxiety leads to greater information-seeking in response to large changes}

As in Study 2a, we examined whether anxiety altered the impact of signed and absolute market change on information-seeking. First, a linear regression was conducted for each participant to predict WTP from the signed market change and absolute market change and the resulting betas were than compared to zero with a one sample t-test. Again, we found that participants' willingness to pay for information was greater when the market was going up rather than down (signed market change: $\beta=1.15, \mathrm{t}(47)=2.57, \mathrm{p}=0.013$ ) and when there were large changes in the market (absolute market change: $\beta=1.06, \mathrm{t}(47)=2.02, \mathrm{p}=0.049$, same is true when excluding the one outlier: $\beta=1.41, \mathrm{t}(46)=3.51, \mathrm{p}=0.001)$ (Figure 7A).

Next, we investigated whether the influence of these two features on information-seeking was altered by induced anxiety. To that end, we ran two linear regressions to predict the beta coefficients obtained in the previous analysis for signed and absolute market change from changes in SSAI, controlling for age and gender. Similar to what we found for trait anxiety in Study $2 \mathrm{a}$, results showed that induced anxiety increased information-seeking in response to large changes in the environment $\left(\beta=0.266 \pm 0.118, p=0.029, f^{2}=0.104\right.$; the same results are obtained when excluding the one outlier: $\beta=0.20 \pm 0.088, p=0.027, f^{2}=0.109$; Figure 7B). This result does not only conceptually replicate the finding from Study 2 a (with induced 
anxiety instead of trait anxiety), but it additionally provides evidence that anxiety causally increases information-seeking about large changes in the environment.

In addition, we found in Study $2 \mathrm{~b}$ that induced anxiety was associated with valence-dependent information-seeking, that is, increased anxiety was related to increased seeking of positive information $\left(\beta=0.211 \pm 0.101, \mathrm{p}=0.042, f^{2}=0.09\right.$; the same results are obtained when excluding the two outliers: $\beta=0.146 \pm 0.064, p=0.028, f^{2}=0.11$; Figure 7C).

B)

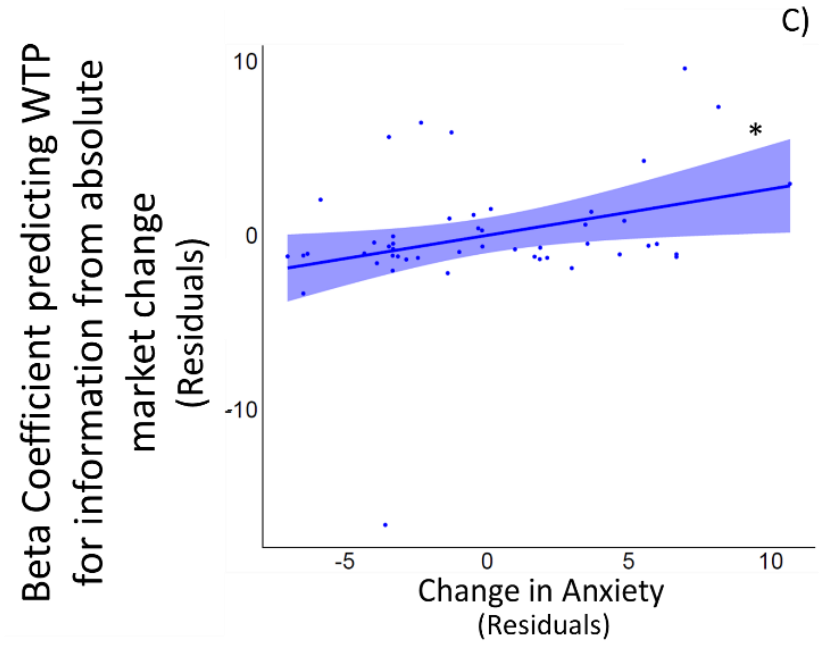

A)

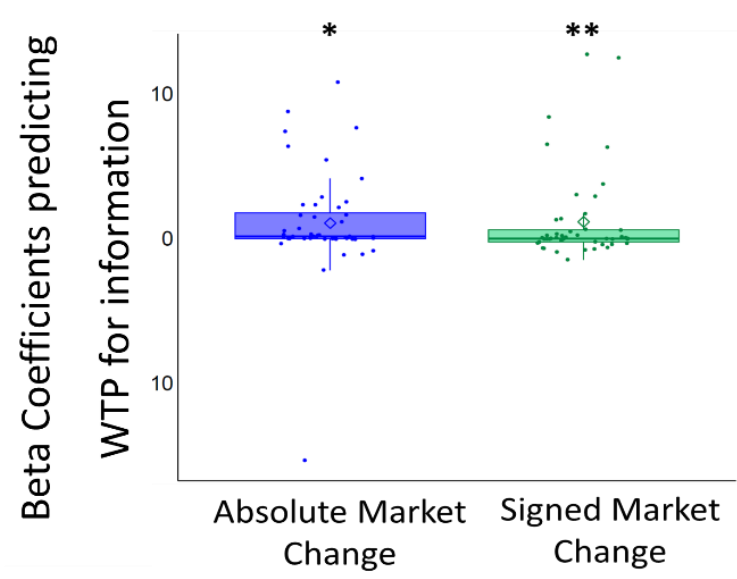

Figure 7. Induced anxiety leads to greater information-seeking in response to large changes (Study 2b). (A) Both signed market change (signaling whether the market was going up or down) and absolute market change (signaling whether there were large or small changes in the market) predicted participants' willingness to pay for information. Plotted are the beta coefficients reflecting the effect of signed (green) and absolute (purple) market change on WTP. Horizontal lines indicate median values, boxes indicate $25-75 \%$ interquartile range, the crosses indicate mean values and whiskers indicate 1.5 $\times$ interquartile range; individual scores are shown separately as circles. $* * p=0.01, * p<0.05$. (B-C) Induced anxiety resulted in greater influence of $(\mathbf{B})$ magnitude of market change $(p=0.029$; results do not change when excluding the one outlier) and $(\mathbf{C})$ valence $(\mathrm{p}=0.042$; results do not change when excluding two outliers) of market change on information-seeking. Plotted are the partial linear regressions predicting the beta coefficients shown in (A) from change in anxiety, controlling for age and gender. 
In sum, the results suggest that anxiety does not alter the overall tendency to seek or avoid information, but rather is associated with greater information-seeking in response to large changes, both in Study 2a (trait anxiety) and Study 2b (induced anxiety), and with greater information-seeking about positive information only in Study $2 b$.

\section{Discussion}

In the present study we shed new light on the relationship between anxiety and informationseeking. We show that anxiety leads to increased information-seeking in response to large changes, rather than to a general increase in the desire for information. This is true even when the cause of the anxiety (e.g., a stressful social situation) is not related to those large changes (e.g., changes are financial). This suggests that the influence of anxiety on information-seeking can "spill over" to other aspects of one's life that are not necessarily related to the source of anxiety.

In addition, results from our ecological study show that participants who reported greater anxiety during the pandemic sought more information about COVID-19, consistent with past studies (e.g., Drouin et al., 2020; Ebrahim et al., 2020; Loosen et al., 2021). While the natural setting of the COVID-19 pandemic is ecologically relevant, inferences from this dataset are limited. Indeed, in the context of the pandemic, many factors that may drive informationseeking are confounded, including large change in the environment and an increase in negatively-valenced news.

We were able to fully dissociate these drives in controlled laboratory experiments. In particular, results from our information-seeking task suggest that participants with higher trait anxiety exhibit a selective increase in information-seeking when large changes in the environment occur. These results were replicated in a second study where anxiety was experimentally induced, providing causal evidence for this relationship. These laboratory results may speak to the findings of our ecological study, suggesting that large change in the environment during the pandemic may have contributed to the large increase in information-seeking in anxious individuals. We note that in the second laboratory study, we also found that anxiety caused increased information-seeking about positive information. This finding is consistent with the idea that under anxiety greater evidence is required to form a positive belief than a negative belief (Garrett et al., 2018; Globig et al., 2020), thus information-seeking may be larger when evidence points in a positive direction. However, as the effect was relatively small and was not conceptually replicated, it should be interpreted with caution.

Heightened information-seeking in response to large changes may be adaptive. When experiencing a major life change (e.g. moving countries, changing jobs, etc), seeking information helps adapt to the new environment. Interestingly, however, in our lab studies, this effect was present even though the cause of the anxiety was unrelated to the information sought. For example, in Study $2 b$, inducing anxiety by exposing participants to a stressful social 
situation, led to greater desire for information about the value of stocks when the financial market was experiencing large changes. This finding may reflect a compensatory mechanism. In particular, anxious individuals have difficulty learning when in volatile environments (Browning et al., 2015; Hein et al., 2021) and generally misestimate the levels of risk and uncertainty when learning and making decisions (Charpentier et al., 2017; Pulcu \& Browning, 2019). They may thus require more information to adapt their behavior in changing environment and reduce uncertainty. This tendency, however, may become maladaptive if the information is predominantly negative, leading to low mood (Johnston \& Davey, 1997; Soroya et al., 2021; Shabahang et al., 2020; Dörnemann et al., 2021), as well as increased stress and psychopathology symptoms (Norr et al., 2014; Garfin et al., 2020; Price at al., 2021).

Previous studies investigating information-seeking and psychopathology suggest that different clinical populations may be characterized by different information-seeking patterns. For example, it has been suggested that individuals who suffer from social anxiety disorders look for less information before making social ranking decisions (Aderka et al., 2013) while high obsessive-compulsive individuals seek more information in general (Loosen et al, 2021) and need more evidence to make decisions (Hauser et., 2017). The present work, while not focused on clinical populations, raises the interesting possibility that information-seeking behaviors in highly changing environments may be altered in clinical anxiety. Specifically, clinically anxious individuals may seek a large amount of information in highly changing environments, in ways that become maladaptive, for example by leading to an inability to make decisions. Future investigations of information-seeking in clinical anxiety are warranted to investigate such relationship.

Taken together, our findings shed new light on the intricate relationship between anxiety and information-seeking by showing a tight link between anxiety and increased informationseeking in response to large changes. When switching jobs or moving cities, anxiety may drive individuals to search for more information, including information unrelated to the cause of the anxiety. On one hand, this may reduce uncertainty and increases adaptation, but in extreme cases may lead to indecision and information overload.

\section{Data and Code availability}

Anonymized data and code will be available at a dedicated Github repository upon publication.

\section{Author Contributions}

C.J.C., I.C.D., V.V. and T.S. developed the study concept. C.J.C., I.C.D., V.V., L.G. and T.S. contributed to the study design. Data collection was performed by C.J.C., M.G. and L.G. Data analysis and interpretation were performed by C.J.C., I.C.D., V.V. and T.S. All authors drafted the manuscript and approved the final version of the manuscript for submission. 


\section{Acknowledgments}

We thank Bastien Blain, Moshe Glickman, Christopher Kelly, Gaia Molinaro, Christina Maher, Liron Rozenkrantz and Sarah Zheng for providing comments. The work was funded by a Wellcome Trust Senior Research Fellowship to TS, by a FWO postdoctoral fellowship to ICD and by a Sir Henry Wellcome Postdoctoral Fellowship (218642/Z/19/Z) to CJC. The authors declare no competing financial interests.

\section{References}

1. Aderka, I. M., Haker, A., Marom, S., Hermesh, H., \& Gilboa-Schechtman, E. (2013). Information-seeking bias in social anxiety disorder. Journal of abnormal psychology, 122(1), 7.

2. Bar-Haim Y, Lamy D, Pergamin L (2007) Threat-related attentional bias in anxious and nonanxious individuals: a meta-analytic study. Psychol Bull 133:1-24.

3. Baumgartner, S. E., \& Hartmann, T. (2011). The role of health anxiety in online health information search. Cyberpsychology, behavior, and social networking, 14(10), 613618.

4. Becker, G. M., Degroot, M. H. \& Marschak, (1964) J. Measuring utility by a singleresponse sequential method. Behav. Sci. 9, 226-232

5. Birkett, M.A. (2011). The Trier Social Stress Test Protocol for Inducing Psychological Stress. 754 J. Vis. Exp.

6. Blain, B., Globig, L. K., \& Sharot, T. (2021). Income shock increases delay discounting independently of emotion. PsyArXiv

7. Browning, M., Behrens, T. E., Jocham, G., O'reilly, J. X., \& Bishop, S. J. (2015). Anxious individuals have difficulty learning the causal statistics of aversive environments. Nature neuroscience, 18(4), 590-596.

8. Charpentier, C. J., De Martino, B., Sim, A., L., Sharot, T., Roiser, J. P. (2016). Emotioninduced loss aversion and striatal-amygdala coupling in low-anxious individuals. Soc. Cogn. Affect. Neurosci. 11(4), 569-579.

9. Charpentier, C. J., Aylward, J., Roiser, J. P., \& Robinson, O. J. (2017). Enhanced Risk Aversion, But Not Loss Aversion, in Unmedicated Patholiogical Anxiety. Biological Psychiatry, 81(12), 1014-1022.

10. Charpentier, C. J., Bromberg-Martin, E. S., \& Sharot, T. (2018). Valuation of knowledge and ignorance in mesolimbic reward circuitry. Proceedings of the National Academy of Sciences, 115(31), E7255-E7264.

11. Cisler JM, Koster EHW (2010) Mechanisms of attentional biases towards threat in anxiety disorders: An integrative review. Clin Psychol Rev 30:203-216.

12. Dillard J. P., Li, R., Yang, C. (2020). Fear of Zika: Information Seeking as a Cause and Consequence. Health Communication. DOI: 10.1080/10410236.2020.1794554

13. Dörnemann, A., Boenisch, N., Schommer, L., Winkelhorst, L., \& Wingen, T. (2021). How do Good and Bad News Impact Mood During the Covid-19 Pandemic? The Role of Similarity. https://doi.org/10.31219/osf.io/sy2kd 
14. Drouin, M., McDaniel, B. T., Pater, J., \& Toscos, T. (2020). How parents and their children used social media and technology at the beginning of the COVID-19 pandemic and associations with anxiety. Cyberpsychology, Behavior, and Social Networking, 23(11), 727-736.

15. Ebrahim, A. H., Saif, Z. Q., Buheji, M., AlBasri, N., Al-Husaini, F. A., \& Jahrami, H. (2020). COVID-19 information-seeking behavior and anxiety symptoms among parents. OSP Journal of Health Care and Medicine, 1(1), 1-9.

16. Fiocco, A.J., Joober, R., \& Lupien, S.J. Education modulates cortisol reactivity to the Trier Social Stress Test in middle-aged adults. Psychoneuroendocrinology. 32, 11581163 (2007).

17. Fries, E., Hellhammer, D.H., \& Hellhammer, J. (2006). Attenuation of the hypothalamic-pituitary-adrenal axis responsivity to the Trier Social Stress Test by the benzodiazepine alprazolam. Psychoneuroendocrinology. 31, 1278-1288.

18. Fung, B. J., Qi., S., Hassabis, D., Daw, N., \& Mobbs, D. (2019) Slow escape decisions are swayed by trait anxiety. Nature Human Behaviour, 3, 702-708.

19. Gadarian, S. K., \& Albertson, B. (2014). Anxiety, immigration, and the search for information. Political Psychology, 35(2), 133-164.

20. Gallagher, M. W., Naragon-Gainey, K., \& Brown, T. A. (2014). Perceived control is a transdiagnostic predictor of cognitive-behavior therapy outcome for anxiety disorders. Cognitive therapy and research, 38(1), 10-22.

21. Garfin, D. R., Cohen Silver, R., \& Holman, E. A. (2020). The Novel Coronavirus (COVID-2019) Outbreak: Amplification of Public Health Consequences by Media Exposure. Health Psychology, 39(5), 355-357.

22. Garrett, N., González-Garzón, A. M., Foulkes, L., Levita, L., \& Sharot, T. (2018). Updating beliefs under perceived threat. Journal of Neuroscience, 38(36), 7901-7911.

23. Globig, L. K., Witte, K., Feng Jones, G., \& Sharot, T. (2021). Under threat weaker evidence is required to reach undesirable conclusions. Journal of Neuroscience, 41(30), 6502-6510.

24. Globig, L.K., Blain, B., \& Sharot, T. (2020). When Private Optimism meets Public Despair: Dissociable effects on behavior and well-being. PsyArXiv. https://doi.org/10.31234/osf.io/gbdn8

25. Hauser, T. U., Moutoussis, M., Iannaccone, R., Brem, S., Walitza, S., Drechsler, R., ... \& Dolan, R. J. (2017). Increased decision thresholds enhance information gathering performance in juvenile Obsessive-Compulsive Disorder (OCD). PLoS Computational Biology, 13(4), e1005440.

26. Hein, T. P., de Fockert, J., Herrojo Ruiz, M. (2021) State anxiety biases estimates of uncertainty and impairs reward learning in volatile environments. NeuroImage, 224, 117424.

27. Huang, H., Thompson, W., Paulus, M.P. (2017). Computational Dysfunctions in Anxiety: Failure to Differentiate Signal From Noise. Biol Psychiatry, 15;82(6):440446.

28. Johnston, W. M., \& Davey, G. C. L. (1997). The psychological impact of negative TV news bulletins: The catastrophizing of personal worries. British Journal of Psychology, 88, 85-91. 
29. Kirschbaum, C., Pirke, K. M., \& Hellhammer, D. H. (1993). The 'Trier Social Stress Test'-a tool for investigating psychobiological stress responses in a laboratory setting. Neuropsychobiology, 28(1-2), 76-81.

30. Kirschbaum, C., Würst, S., \& Hellhammer, D. (1992) Consistent sex differences in cortisol responses to psychological stress. Psychosom. Med. 54, 648-657.

31. Kudielka, B.M., Hellhammer, D.H., \& Kirschbaum, C. (2007) Ten years of research with the Trier Social Stress Test - Revisited. Social Neuroscience: Integrating Biological and Psychological Explanations. Harmon-Jones, E., Winkielman, P., Eds. Guilford Press, New York.

32. Loosen, A. M., Skvortsova, V., \& Hauser, T. U. (2021). Obsessive-compulsive symptoms and information seeking during the Covid-19 pandemic. Translational psychiatry, 11(1), 1-10.

33. MacLeod C, Mathews A (1988) Anxiety and the allocation of attention to threat. Q J Exp Psychol Sect A Hum Exp Psychol 40:653-670.

34. Marteau, T.M.; Bekker, H. The development of a six-item short-form of the state scale of the Spielberger State-Trait Anxiety Inventory (STAI). Br. J. Clin. Psychol. 1992, 31, 301-306.

35. McMullan, R. D., Berle, D., Arnáez, S., \& Starcevic, V. (2019). The relationships between health anxiety, online health information seeking, and cyberchondria: Systematic review and meta-analysis. Journal of affective disorders, 245, 270-278.

36. Norr, A. M., Capron, D. W., \& Schmidt, N. B. (2014). Medical information seeking: Impact on risk for anxiety psychopathology. Journal of Behavior Therapy and Experimental Psychiatry, 45, 402-407.

37. Oswald, L.M., Zandi, P., Nestadt, G., Potash, J.B., Kalaydjian, A.E., \& Wand, G.S. (2006) Relationship between cortisol responses to stress and personality. Neuropsychopharmacology. 31, 1583-1591.

38. Price, M., Legrand, A. C., Brier, Z. M., van Stolk-Cooke, K., Peck, K., Sheridan Dodds, P., ... \& Adams, Z. W. (2021). Doomscrolling during COVID-19: The negative association between daily social and traditional media consumption and mental health symptoms during the COVID-19 pandemic. https://doi.org/10.31234/osf.io/s2nfg

39. Pulcu, E., \& Browning, M. (2019). The misestimation of uncertainty in affective disorders. Trends in Cognitive Sciences, 23(10), 865-875.

40. Shabahang, R., Aruguete, M. S., \& McCutcheon, L. E. (2020). Online health information utilization and online news exposure as predictor of COVID-19 anxiety. N Am J Psychol, 22(3), 469-482.

41. Sharot, T., \& Sunstein, C. R. (2020). How people decide what they want to know. Nature Human Behaviour, 4(1), 14-19.

42. Soroya, S.H., Farooq, A., Mahmood, K., Isoaho, J., Zara, S.-e. (2021). From information seeking to information avoidance: Understanding the health information behavior during a global health crisis. Information Processing and Management, 58, 102440 .

43. Vellani, V., de Vries, L. P., Gaule, A., \& Sharot, T. (2020). A selective effect of dopamine on information-seeking. Elife, 9, e59152. 
44. Vogel, S., Kluen, L. M., Fernández, G., \& Schwabe, L. (2018). Stress affects the neural ensemble for integrating new information and prior knowledge. NeuroImage, 173, 176187.

45. Xu, P., Gu, R., Broster, L. S., Wu, R., Van Dam, N. T., Jiang, Y., Fan, J., \& Luo, Y. J. (2013). Neural basis of emotional decision making in trait anxiety. J. Neurosci., 33, 18641-18653. 


\section{Supplementary Materials}

\section{Methods}

\section{Additional information collected in the questionnaire at Time Point 1}

These measures are part of parallel studies conducted in our lab (Globig et al., 2020; Blain et al., 2021):

COVID-19-related Information-seeking: Participants were asked to indicate how often they consumed information on COVID-19 (news, internet etc...) on the following scale: 1 (Never), 2 (Less than once a week), 3 (At least once a week), 4 (Once a day), 5 (At least 4 times per day), 6 (At least once an hour).

Demographics: Participants were asked to indicate their age, gender, ethnicity, current place of residence, level of education, household income, health insurance satisfaction, political orientation and whether they had children.

Covid-19 related anxiety: Participants who declared to have children also answered to the following question "Are you anxious about homeschooling in light of COVID-19?" on a continuous visual analogue scale ranging from 0 (none at all) to 100 (very much). This question has not been included in the main analysis as only the $22.36 \%$ of subjects indicated to have children.

Impact of COVID-19 on income: Participants were asked to indicate what was the impact of COVID-19 on their income on a scale from 1 (None) to 6 (Extremely high).

Happiness: In order to assess happiness, participants were asked the following questions: (i)"Taken all together, how happy are you with your life these days? Mark your rating relative to the least and most happy time of your life." Participants were asked to respond on a continuous visual analogue scale ranging from 0 (least happy time of your life) to 100 (most happy time of your life). (ii) "Think about right now. How happy are you at this moment?". Participants were asked to respond on a continuous visual analogue scale ranging from 0 (very unhappy) to 100 (very happy).

Emotions: Participants were asked to report whether in the last 24 hours they felt fear, hopeful, joyful, sad, angry and surprised on a continuous scale ranging from "Not at all" to "Very much".

Optimism about getting COVID-19: Participants were asked to indicate: "Relative to others of your age and gender do you think you are less/more likely to get COVID-19?" on a scale from 1 (much less likely) to 5 (much more likely).

Perceived danger to humanity: Participants were asked to indicate: "Do you think COVID19 presents a real danger to the health of the human population?" on a continuous visual analogue scale from 0 (not really) to 100 (extreme danger).

Compliance measures: Participants rated on a continuous visual analogue scale ranging from 0 (none at all) to 100 (a lot): a) "How much effort do you make to wash your hands regularly?"; b) "How much effort do you make to socially distance yourself from others?"; c) "How much effort do you make to avoid touching your face?", as well on a continuous visual analogue scale ranging from 0 (zero) to 100 (many times) d) "In the past week how many times have 
you been to another person's house ?"; e) How many days this week have you been closer than 1 meter to another person (except those you live with)?".

Sense of agency: Participants completed a questionnaire assessing sense of agency which was composed by 2 parts assessing personal mastery and perceived constraints. (Lachman \& Weaver, 1998).

Behavioral Changes: To assess behavioral changes caused by the pandemic participants were asked to indicate the frequency of face-to-face interaction, online/telephone interaction, physical activity, outdoor activity, visiting places of religious worship, social connectedness before and after the restrictions. Behaviors were reported on the same scales for before and after the restrictions.

Addictive behaviors: Participants reported frequency of habitudinal and addictive behaviors including smoking, alcohol consumption, gambling, eating before and after the restrictions.

Psychopathology: Participants completed the: Obsessive-Compulsive Inventory - Revised (OCI-R, Foa, Kozak, Salkovskis, Coles, \& Amir, 1998), Patient Health Questionnaire (PHQ9, Kroenke, Spitzer, \& Williams, 2001), Apathy Evaluation Scale (AES, Mann, 1990), Life Orientation Optimism Test (LOT-R, Molina et al., 2013).

Psychosocial Questionnaires : Participants also completed a series of psychosocial questionnaires assessing empathic concern (Davis, 1983), resilience (Smith et al., 2008), narcissism (Leckelt et al., 2018), risk-taking propensity (GRiPS, Zhang, Highhouse, \& Nye, 2019).

Stress Coping: Participants responded on a 5-point Likert Scale from 1 (strongly disagree) to 5 (strongly agree) to the following items: 1) The effects of stress are negative and should be avoided. 2) The effects of stress are positive and should be utilized.

Health Anxiety: Participants were asked to indicate their health anxiety on a 5-point Likert Scale from 1 (very inaccurate) to 5 (very accurate): Often I am concerned about diseases I might have.

Non-Conformity: Participants were asked to indicate conformity on a 5-point Likert Scale from 1 (very strong disagreement) to 5 (very strong agreement): I prefer to make my own way in life rather than find and follow.

Social Support and Connectedness: Participants were asked to indicate on a 7-point Likert Scale from 1 (strongly disagree) to 7 (strongly agree): My friends/family give me the support I need. We presented participants with a modified version of the "inclusion of others in the self" scale (Aron, Aron, Tudor, \& Nelson, 1991).

Behavioral Economic Tasks: Participants completed a series of established behavioral tasks including a one-shot dictator game (Kahneman, 1986), an intertemporal choice task (Kirby \& Maraković, 1996) and a loss aversion task (Rutledge, Skandali, Dayan, \& Dolan, 2014).

\section{Additional information collected in the questionnaire at Time Point 2 (which} are part of parallel studies conducted in our lab):

Many of the items presented in the survey completed by subjects in Time Point 1 for the parallel studies conducted in the lab were not included At Time Point 2. Questions included at Time Point 2 are: happiness, optimism about getting COVID-19, compliance, emotions, behavioral changes, impact of COVID-19 on income, sense of agency. 
In Time Point 2 the following changes were made:

- The question "Do you think COVID-19 presents a real danger to the health of the human population?" was changed to "How likely are people to get COVID-19?" on a scale from 1 (extremely unlikely) to 5 (extremely likely)". 500 of our participants also completed the original question.

- A question to assess absolute general expectations about getting COVID-19 was added by asking participants "How likely do you think you are to get COVID-19?" on a scale from 1 (extremely unlikely) to 5 (extremely likely)".

\section{Attention catch trials}

Time point 1

In order to check participants' engagement and attention, 10 catch trials were inserted in the survey. In the catch trials subjects were asked to select a specific answer (for example: Please select 'strongly disagree'). Specifically, 2 catch trials were present in the intertemporal choice block, 1 in the loss aversion block and one in each of the blocks assessing the following constructs: risk taking, perceived constraints, personal mastery, obsessive-compulsive behaviors, resilience, optimism and apathy. Participants who failed to select the correct answer more than once were excluded from analysis $(\mathrm{N}=21)$.

Time point 2

2 catch trials were present, specifically in the blocks assessing perceived constraints and personal mastery. Participants who failed to select the correct answer more than once were excluded from analysis $(\mathrm{N}=0)$.

\section{Additional Information on the task}

In the last two blocks of the task (blocks 3-4), participants indicated what they expected the change in portfolio value to be on that trial from -4 ('decreased a lot') to +4 ('increased a lot') and their confidence in that rating from 1 ('not confident at all') to 9 ('extremely confident'). These ratings occurred directly after they observed the global market on each trial. Each rating had a 8-s time limit. This was followed by the WTP scale and delivery of information or no information as in previous blocks.

\section{Supplementary References}

1. Blain, B., Globig, L. K., \& Sharot, T. (2021). Income shock increases delay discounting independently of emotion.

2. Aron, A., Aron, E. N., Tudor, M., \& Nelson, G. (1991). Close relationships as including other in the self. Journal of personality and social psychology, 60(2), 241.

3. Blain, B., Globig, L. K., \& Sharot, T. (2021). Income shock increases delay discounting independently of emotion. PsyArXiv

4. Davis, M. H. (1983). Empathic concern and the muscular dystrophy telethon: Empathy as a multidimensional construct. Personality and Social Psychology Bulletin, 9(2), 223-229. 
5. Engel, C. (2011). Dictator games: A meta study. Experimental economics, 14(4), 583610.

6. Foa, E. B., Kozak, M. J., Salkovskis, P. M., Coles, M. E., \& Amir, N. (1998). The validation of a new obsessive-compulsive disorder scale: The Obsessive-Compulsive Inventory. Psychological Assessment, 10(3), 206.

7. Globig, L.K., Blain, B., \& Sharot, T. (2020). When Private Optimism meets Public Despair: Dissociable effects on behavior and well-being. PsyArXiv

8. Kirby, K. N., \& Maraković, N. N. (1996). Delay-discounting probabilistic rewards: Rates decrease as amounts increase. Psychonomic bulletin \& review, 3(1), 100-104.

9. Kroenke, K., Spitzer, R. L., \& Williams, J. B. (2001). The PHQ-9: validity of a brief depression severity measure. Journal of general internal medicine, 16(9), 606-613.

10. Lachman, M. E., \& Weaver, S. L. (1998). The sense of control as a moderator of social class differences in health and well-being. Journal of personality and social psychology, 74(3), 763.

11. Leckelt, M., Wetzel, E., Gerlach, T. M., Ackerman, R. A., Miller, J. D., Chopik, W. J., ... \& Back, M. D. (2018). Validation of the Narcissistic Admiration and Rivalry Questionnaire Short Scale (NARQ-S) in convenience and representative samples. Psychological assessment, 30(1), 86.

12. Mann, R. S. (1990). Differential diagnosis and classification of apathy. Am J Psychiatry, 147(1), 22-30.

13. Molina, K. M., Molina, K. M., Goltz, H. H., Kowalkouski, M. A., Hart, S. L., Latini, D., ... Gidron, Y. (2013). Revised Life Orientation Test (LOT-R). Encyclopedia of Behavioral Medicine, 1678- 1678.

14. Rutledge, R. B., Skandali, N., Dayan, P., \& Dolan, R. J. (2014). A computational and neural model of momentary subjective well-being. Proceedings of the National Academy of Sciences, 111(33), 12252-12257.

15. Smith, B. W., Dalen, J., Wiggins, K., Tooley, E., Christopher, P., \& Bernard, J. (2008). The brief resilience scale: assessing the ability to bounce back. International journal of behavioral medicine, 15(3), 194-200.

16. Zhang, D. C., Highhouse, S., \& Nye, C. D. (2019). Development and validation of the general risk propensity scale (GRiPS). Journal of Behavioral Decision Making, 32(2), 152-167. 